\title{
A novel tetracycline-responsive transgenic mouse strain for skeletal muscle-specific gene expression
}

\author{
Masahiro Iwata ${ }^{1,3,4+}$, Davis A. Englund ${ }^{1,2+}$, Yuan Wen ${ }^{1,3}$, Cory M. Dungan ${ }^{1,2}$, Kevin A. Murach ${ }^{1,2}$, Ivan J. Vechetti Jr $r^{1,3}$, \\ Christopher B. Mobley ${ }^{1,3}$, Charlotte A. Peterson ${ }^{1,2}$ and John J. McCarthy ${ }^{1,3^{*}}$
}

\begin{abstract}
Background: The tetracycline-responsive system (Tet-ON/OFF) has proven to be a valuable tool for manipulating gene expression in an inducible, temporal, and tissue-specific manner. The purpose of this study was to create and characterize a new transgenic mouse strain utilizing the human skeletal muscle a-actin (HSA) promoter to drive skeletal muscle-specific expression of the reverse tetracycline transactivator (rtTA) gene which we have designated as the HSA-rtTA mouse.

Methods: To confirm the HSA-rtTA mouse was capable of driving skeletal muscle-specific expression, we crossed the HSA-rtTA mouse with the tetracycline-responsive histone H2B-green fluorescent protein (H2B-GFP) transgenic mouse in order to label myonuclei.

Results: Reverse transcription-PCR confirmed skeletal muscle-specific expression of rtTA mRNA, while single-fiber analysis showed highly effective GFP labeling of myonuclei in both fast- and slow-twitch skeletal muscles. Pax7 immunohistochemistry of skeletal muscle cross-sections revealed no appreciable GFP expression in satellite cells.

Conclusions: The HSA-rtTA transgenic mouse allows for robust, specific, and inducible gene expression across muscles of different fiber types. The HSA-rtTA mouse provides a powerful tool to manipulate gene expression in skeletal muscle.
\end{abstract}

Keywords: Skeletal muscle-specific, Tetracycline-responsive

\section{Background}

Since the original description, the tetracycline-responsive system (Tet-ON/OFF) has proven to be a powerful tool in biomedical research because of the ability to manipulate gene expression within the mouse in both a temporal and tissue-specific manner [1, 2]. Although a number of skeletal muscle-specific Tet-ON/OFF mice have been described, they have used promoters that drive primarily fast-twitch, type II gene expression; in addition, these mice are not readily available $[3,4]$. To address these limitations,

\footnotetext{
*Correspondence: jjmcca2@uky.edu

${ }^{+}$Masahiro Iwata and Davis A. Englund contributed equally to this work.

'The Center for Muscle Biology, University of Kentucky, Lexington, KY 40536, USA

${ }^{3}$ Department of Physiology, College of Medicine, University of Kentucky, 800 Rose Street, Medical Science Building, Rm: MS-607A, Lexington, KY 40536, USA

Full list of author information is available at the end of the article
}

we generated a transgenic mouse which uses the human skeletal muscle $\alpha$-actin (HSA) promoter to drive skeletal muscle-specific expression of the reverse-tetracycline transactivator (rtTA) which we have designated as the HSA-rtTA mouse. To validate the HSA-rtTA mouse, we crossed it with the tetracycline-responsive histone $\mathrm{H} 2 \mathrm{~B}$ green fluorescent protein (TRE-H2B-GFP) mouse to easily visualize and quantify myonuclear GFP expression following doxycycline treatment [5]. As expected, rtTA mRNA was highly expressed in skeletal muscle as $>95 \%$ of myonuclei were GFP-positive in both type I and type II muscles. Importantly, an extremely small number of satellite cells appeared to be GFP-positive in soleus muscle cross-section, thus confirming the ability of the HSA-rtTA mouse to drive robust skeletal muscle-specific expression of a tetracycline-responsive gene of interest.

(c) The Author(s). 2018 Open Access This article is distributed under the terms of the Creative Commons Attribution 4.0 International License (http://creativecommons.org/licenses/by/4.0/), which permits unrestricted use, distribution, and reproduction in any medium, provided you give appropriate credit to the original author(s) and the source, provide a link to the Creative Commons license, and indicate if changes were made. The Creative Commons Public Domain Dedication waiver (http://creativecommons.org/publicdomain/zero/1.0/) applies to the data made available in this article, unless otherwise stated. 


\section{Methods}

\section{Generating the HSA-rtTA transgenic mouse}

As previously described by us for the HSA-MerCreMer transgene, the promoter and first exon $(-2,000$ to +239 relative to the transcription start site) of the human skeletal muscle $\alpha$-actin (HSA) gene was amplified from human genomic DNA (Promega, Madison, WI, USA) and cloned into the ClaI site of the SG5 expression vector (Agilent Technologies, Santa Clara, CA, USA) upstream of the $\beta$-globin intron II [6]. The rtTA cDNA was amplified from the pCMV-Tet3G expression vector (Takara Bio, Mountain View, CA, USA) and cloned into the EcoRI/BamHI sites of the pSG5-HSA plasmid to generate the pSG5-HSA-rtTA; the rtTA insert was subsequently sequenced for verification. The HSA-rtTA transgene (Fig. 1) was released from the plasmid by HindIII/NsiI enzyme digestion, gel-purified using the QIAquick Gel Extraction Kit according to the manufacturer's directions (Qiagen, Valencia, CA, USA), and then provided to the University of Michigan Transgenic Animal Model Core for microinjection. F1 generation pups were screened by PCR for the presence of the rtTA sequence using genomic DNA isolated from tail snips with the following primers: F, 5'ATGTCTAGACTGGACAAG AGCA AAG-3'; R, 5'-TTACCCGGGGAGCATGTC-3' generating a product of $747 \mathrm{bp}$. Eight F1 pups were positive for the HSA-rtTA transgene and subsequently crossed to the TRE-H2B-GFP mouse (The Jackson Laboratory, stock number 005104) to determine the ability to drive H2B-GFP expression following doxycycline treatment. Of the eight founder lines, line 6 was identified as driving robust H2B-GFP expression in both slow- and fast-twitch muscles of the lower hind limbs and was further characterized as described below. For convenience, the HSA-rtTA/TRE-H2B-GFP mouse is referred to as the HSA-GFP mouse.

\section{Doxycycline treatment}

To induce H2B-GFP expression, 3-10-month-old HSAGFP mice were administered doxycycline $(0.5 \mathrm{mg} / \mathrm{mL})$ in drinking water supplemented with $2 \%$ sucrose for 3 weeks. Tissue was collected immediately upon completion of doxycycline treatment. To determine the earliest time of GFP induction, skeletal muscle was collected after $12 \mathrm{~h}$ or $24 \mathrm{~h}$ following doxycycline administration.

\section{Analysis of rtTA gene expression}

Total RNA was isolated from skeletal muscles (gastrocnemius, plantaris, soleus, extensor digitorum longus (EDL), tibialis anterior (TA), diaphragm and heart, and nonmuscle tissue (brain, liver, lung, stomach, spleen, kidney, and fat) of HSA-GFP mice. Tissue was immediately frozen in liquid nitrogen upon excision and subsequently homogenized using a Bullet Blender (Next Advance Inc., Averill Park, NY, USA) in Direct-zol (Zymo Research, Irvine, CA, USA) according to the manufacturer's instructions. Total RNA concentration and quality were determined by nanoVue spectrophotometer (GE Healthcare, USA). cDNA was synthesized from $1 \mu \mathrm{g}$ of total RNA using the SuperScript ${ }^{\circ}$ VILO IV ${ }^{\mathrm{Tm}}$ (ThermoFisher Scientific, Waltham, MA, USA) according to the manufacturer's instructions. PCR analysis of rtTA mRNA accumulation used the following primers: F, 5' - GAGGAACAGGAGC ATCAAGTAG-3'; R, 5' - GT CAGCAGGCAGCATATCA-3' and generated a 270 bp product.

\section{Single fiber analysis}

GFP + and GFP - myonuclei were counted on isolated single muscle fibers as previously described by us [7]. Briefly, hind limb muscles were fixed in situ at resting length in $4 \%$ paraformaldehyde for $48 \mathrm{~h}$. Fixed whole muscles were removed from the hind limb, dissected, and dissociated in $40 \%$ sodium hydroxide with manual manipulation at room temperature. Isolated fibers were then stained with DAPI and carefully pipetted on to glass slides and covered using Vectashield (Vector Laboratories, Burlingame, CA, USA).

\section{Immunohistochemistry}

For immunohistochemistry (IHC) analyses, the various hind limb muscles were covered in Tissue-Tek optimal cutting temperature compound (Sakura Finetek, Torrance, CA, USA) and pinned at resting length to a cork covered in aluminum foil. Muscles were frozen in liquid nitrogen-cooled isopentane and stored at $-80{ }^{\circ} \mathrm{C}$. Muscles were sectioned at the mid-belly on a cryostat at $23{ }^{\circ} \mathrm{C}$. Frozen muscle sections $(7 \mu \mathrm{m})$ were air-dried for at least $1 \mathrm{~h}$ and stored at $-20^{\circ} \mathrm{C}$. For Pax7/DAPI IHC, muscles were first fixed in $4 \%$ paraformaldehyde for $7 \mathrm{~min}$ and then subjected to epitope retrieval. Following

\section{HSA promoter

Fig. 1 A schematic of the HSA-rtTA transgene. The promoter and first exon $(-2,000$ to +239 relative to the transcription start site) of the human skeletal muscle a-actin (HSA) gene regulates expression of an optimized reverse tetracycline transactivator (rtTA) gene which has been reported to be sevenfold more active and 100-fold more doxycycline sensitive than the original Tet-On system [8]. The $\beta$-globin intron II (BGI) and poly(A) tail were incorporated into the transgene to ensure proper splicing and transcript stability, respectively. The positions of the PCR primers used for genotyping are indicated by half-arrows 
epitope retrieval in sodium citrate $(10 \mathrm{mM}, \mathrm{pH}$ 6.5) for $20 \mathrm{~min}$ at $92^{\circ} \mathrm{C}$, endogenous peroxidases were blocked for 7 min with 3\% hydrogen peroxide in phosphate-buffered saline (PBS), followed by $1 \mathrm{~h}$ with $1 \%$ Tyramide Signal Amplification (TSA) blocking reagent (TSA kit, T20935, Invitrogen) supplemented with Mouse-on-Mouse (MoM) IgG blocking reagent (Vector Laboratories, Burlingame, CA, USA). Sections were washed in PBS and incubated overnight with mouse anti-Pax7 IgG1 antibody (1:100, Developmental Studies Hybridoma Bank (DSHB), Iowa City, IA, USA) and chicken anti-GFP antibody (1:200, Abcam, Cambridge, MA, USA) diluted in 1\% TSA blocking reagent. It was necessary to use anti-GFP antibody to detect GFP expression because the antigen retrieval process quenched the GFP signal. The following day, sections were washed with PBS, incubated for $70 \mathrm{~min}$ in goat anti-mouse IgG1 biotinylated secondary antibody (1:1000, 115-065-205, Jackson ImmunoResearch, West Grove, PA, USA) and anti-chicken GFP secondary antibody, (1:250, Abcam), washed in PBS, incubated for $1 \mathrm{~h}$ in streptavidin-horseradish peroxidase (1:500, S-911, Invitrogen) diluted in PBS, washed again in PBS, then incubated for $15 \mathrm{~min}$ in TSA Alexa Fluor 594 (1:500, TSA kit, Invitrogen) in the supplied amplification diluents. Sections were stained with DAPI (1:10,000 in PBS, D35471, Invitrogen) for 5 min and mounted with VectaShield fluorescent mounting media.

\section{Image acquisition and quantification}

GFP+/DAPI+ and GFP-/DAPI+ myonuclei from 10 isolated fibers from four doxycycline-treated mice (two male and two female) were counted for each muscle, resulting in a range of 202-452 myonuclei being analyzed across muscles. Twelve to 15 single fibers from two untreated mice ( 1 male and 1 female) were analyzed for each muscle, resulting in a range of 254-600 myonuclei being analyzed across muscles. For the time course analysis, 35 diaphragm fibers $(n=2)$ were counted representing a total of 906 myonuclei, whereas 37 plantaris fibers $(n=2)$ were counted representing a total of 1294 myonuclei. For IHC, images were captured at $\times 20$ magnification using a Zeiss upright fluorescent microscope (Zeiss Axio Imager M1, Oberkochen, Germany). Whole muscle sections were obtained using the mosaic function in Zeiss Zen 2.3 imaging software. Satellite cells (Pax7+/DAPI+) and GFP+ satellite cells were identified manually using Zen software tools.

\section{Results}

\section{Skeletal muscle-specific rtTA transgene}

To generate the skeletal muscle-specific rtTA transgene for microinjection, we cloned downstream of the human skeletal muscle $\alpha$-actin (HSA) promoter a third generation rtTA gene that was reported to be sevenfold more active and 100-fold more doxycycline sensitive than the original rtTA [8]. A schematic of the HSA-rtTA transgene is shown in Fig. 1.

\section{Skeletal muscle-specific expression of rtTA mRNA}

We determined by reverse transcription-PCR the expression of rtTA mRNA in several hind limb muscles, the diaphragm, fat, and several other non-muscle organs. As shown in Fig. 2, rtTA mRNA was highly expressed in all of the hind limb muscles examined and to a lesser extent in the diaphragm and heart. As expected, rtTA expression was not detectable in any non-muscle tissues.

\section{Effective labeling of myonuclei in hind limb skeletal muscles}

Having established that rtTA was highly enriched in skeletal muscle, we sought to determine how effective the HSA-rtTA transgene was in driving H2B-GFP expression in response to doxycycline treatment. Given that H2B-GFP is nuclearly localized, we used the percentage of myonuclei that were GFP+ on single fibers as a measure

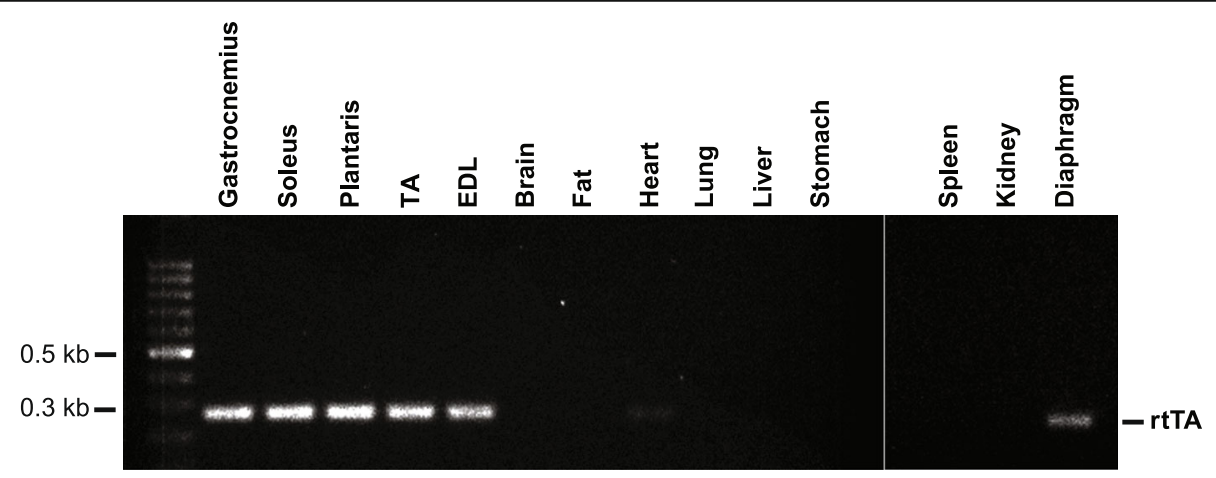

Fig. 2 Skeletal muscle-specific expression of rtTA. PCR analysis of rtTA mRNA expression of different tissues from the HSA-GFP transgenic mouse showed high levels of expression in skeletal muscle (gastrocnemius, soleus, plantaris, tibialis anterior (TA), and extensor digitorum longus (EDL)), modest expression in the diaphragm, very low expression in the heart, and not detectable in non-muscle tissue (brain, fat, lung, liver, stomach, spleen, and kidney) 
of the effectiveness of the HSA-rtTA transgene to induce expression of a tetracycline-responsive gene. Following fixation, single fibers were isolated from the plantaris, gastrocnemius, soleus, tibialis anterior (TA), and extensor digitorum longus (EDL) of doxycycline-treated mice and then stained with DAPI to identify myonuclei. As shown in Fig. 3a, b, greater than 95\% (range of 96.4-97.9\%) of myonuclei were GFP+ across all muscles from
A

Gastrocnemius
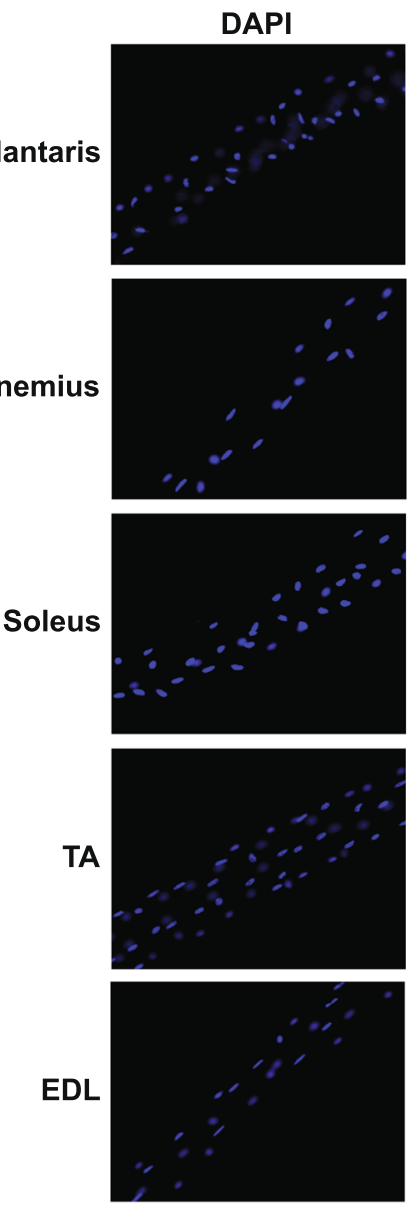

B

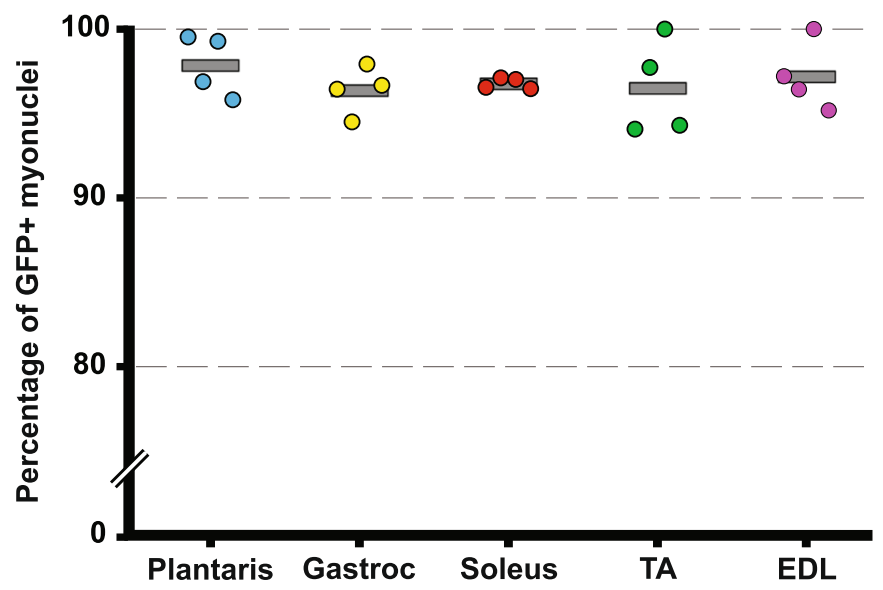

Fig. 3 HSA-rtTA transgene drives robust myofiber expression of tetracycline-responsive H2B-GFP transgene. a Representative single fiber images of hind limb muscles taken from HSA-GFP mice $(n=4)$ treated with doxycycline. Single fiber images show robust myonuclear GFP expression in muscles composed of slow- and fast-twitch fibers. b Quantification of GFP+ myonuclei of single fibers from hind limb skeletal muscles (plantaris, gastrocnemius, soleus, tibialis anterior (TA), and extensor digitorum longus (EDL)) of HSA-GFP mice showed greater than 95\% of all DAPI+ myonuclei within a fiber were GFP+. The gray bar represents the average percentage of GFP-positive myonuclei $(n=4)$ for each muscle 
doxycycline-treated HSA-GFP mice. We observed no GFP + myonuclei in skeletal muscle single fibers of untreated HSA-GFP mice demonstrating tight regulation of tetracycline-responsive H2B-GFP gene (data not shown). These findings confirm the HSA-rtTA mouse is capable of driving robust expression of a tetracycline-responsive gene in adult skeletal muscles composed of both slow- and fast-twitch fibers.
GFP labeling is highly specific to myonuclei

To determine if the HSA-rtTA drove expression of the H2B-GFP transgene in satellite cells, we performed immunohistochemistry on both soleus and plantaris muscle cross-sections for DAPI, Pax7, and GFP. As shown in Fig. 4, GFP labeling did not localize with Pax7 staining; however, in the soleus, of the 190 satellite cells counted, three Pax7+ cells appeared to be GFP+. These results demonstrate the

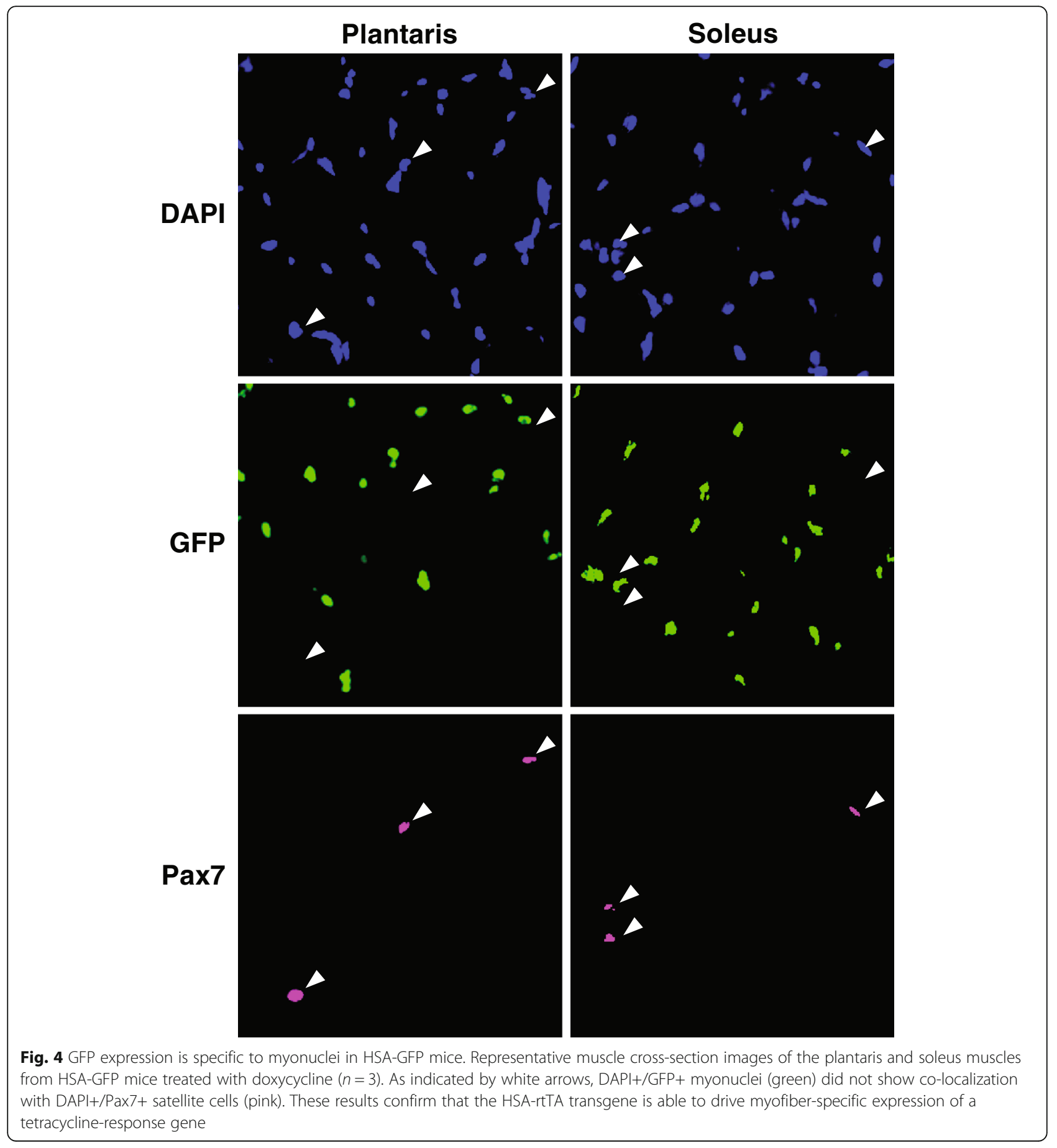


HSA-rtTA drives highly myofiber-specific expression of a tetracycline-responsive transgene.

\section{Rapid GFP labeling of myonuclei}

To determine the time course of GFP labeling of myonuclei, skeletal muscle was collected from HAS-GFP mice after 12 or $24 \mathrm{~h}$ of doxycycline treatment. As shown in Fig. 5a, b, approximately $90 \%$ of myonuclei in the plantaris muscle were GFP-positive after $24 \mathrm{~h}$ of doxycycline treatment; in contrast, GFP expression was not detected following $12 \mathrm{~h}$ of doxycycline administration (data not shown). We also examined whether GFP expression followed the same time course of induction given the modest expression of rtTA mRNA in the diaphragm (see Fig. 2). While GFP expression was not as robust as that observed in the plantaris, $60 \%$ of myonuclei of the diaphragm were GFP-positive following $24 \mathrm{~h}$ of doxycycline treatment, consistent with the lower rtTA mRNA expression (see Fig. 5a-b).

\section{Discussion}

The purpose of this study was to characterize a new skeletal muscle-specific Tet-ON mouse. The human skeletal muscle $\alpha$-actin (HSA) promoter was used to drive skeletal muscle-specific expression of the reversetetracycline transactivator (rtTA), designated as the HSA-rtTA mouse. The HSA promoter contains 2,000 bp of human skeletal $\alpha$-actin 5 '-flanking sequence plus the first exon and $149 \mathrm{bp}$ of the first intron and was first reported by Muscat and Kedes to promote robust, skeletal muscle-specific expression [9]. We choose to use the HSA promoter because we previously showed it was able to drive effective Cre-mediated recombination in both slow- and fast-twitch fibers with minimal expression in the heart [6]. This is an important improvement over a previous skeletal muscle-specific Tet-ON mouse (MCKrtTA) which only allowed over-expression of a gene of interest in fast-twitch, type IIb fibers [10]. Together with the HSA-CreER mouse, the HSA-rtTA mouse now provides the ability to perform loss- and gain-of-function studies, respectively, to determine the in vivo function of a gene of interest in skeletal muscle fibers [6, 11]. The complement to these two inducible, skeletal muscle-specific mice are the satellite cell-specific inducible Cre and Tet-ON mice; however, while the satellite cell-specific Cre mouse has been extensively used, to the best of our
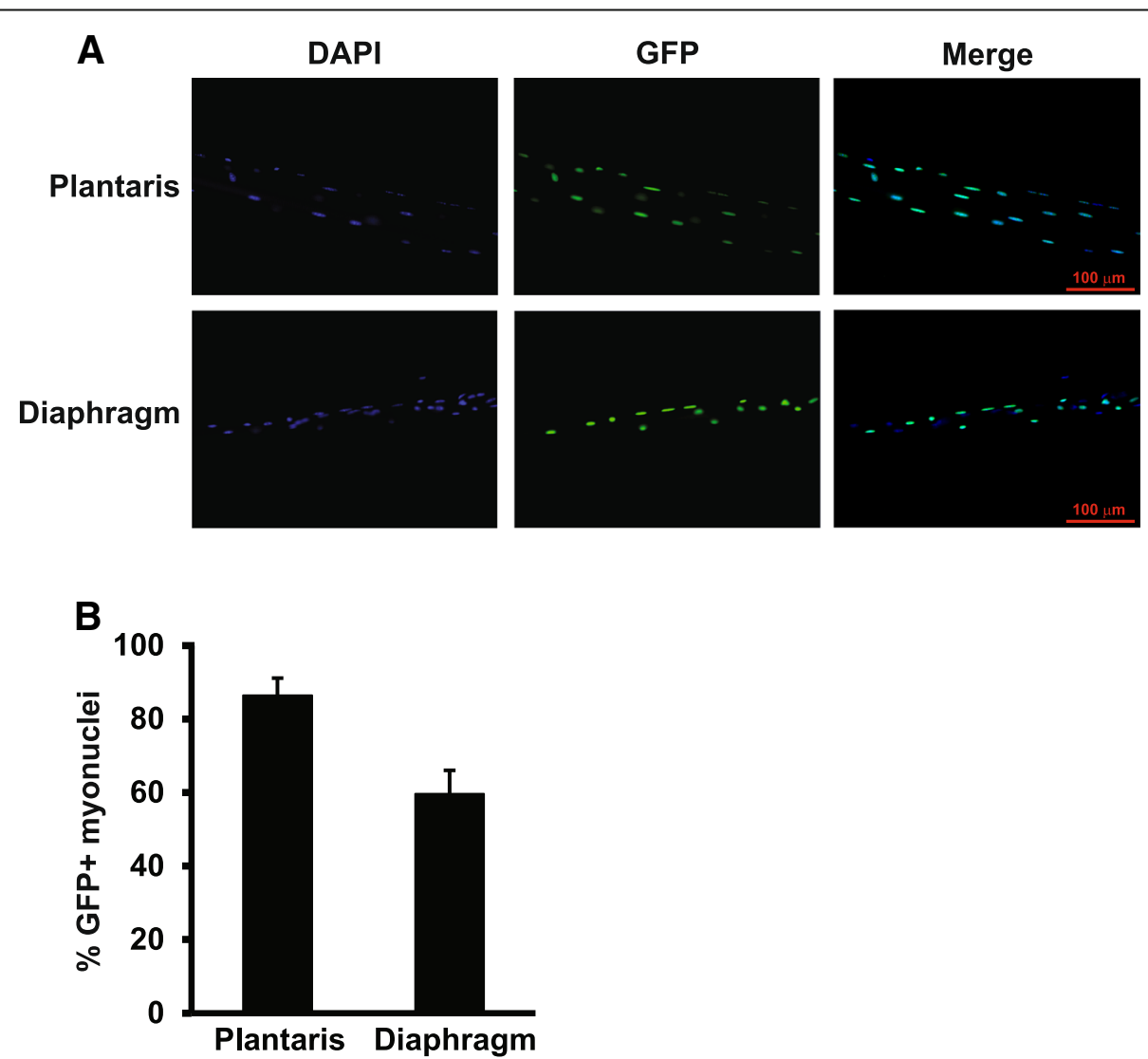

Fig. 5 Rapid GFP labeling of myonuclei. a Representative single fiber images of plantaris and diaphragm muscles taken from HSA-GFP mice ( $n=$ 2) treated with doxycycline for 24 h. $\mathbf{b}$ Quantification of GFP+ myonuclei showed 90\% of myonuclei were GFP-positive in myofibers from the plantaris with $60 \%$ of myonuclei GFP-positive in myofibers isolated from the diaphragm 
knowledge, the satellite cell-specific Tet-ON mouse has yet to be fully characterized [12-15]. Collectively, these inducible, skeletal muscle-, and satellite cell-specific mice provide powerful tools to manipulate in vivo gene expression to identify and better understand the mechanisms regulating skeletal muscle biology in health and disease.

While 3 weeks of doxycycline treatment was able to induce $>95 \%$ GFP labeling of myonuclei, we wanted to know the earliest time point when GFP expression could be detected following doxycycline administration. As shown in Fig. 5, single fiber analysis revealed myonuclear GFP expression was observed as early as $24 \mathrm{~h}$ post-doxycycline exposure; in contrast, we observed no GFP expression at $12 \mathrm{~h}$ post-doxycycline treatment (data not shown). These results demonstrate the HSA-rtTA mouse is very responsive to doxycycline and will provide the ability to study the relative early $(\sim 24 \mathrm{~h})$ effects of gene activation on a given biological process. For example, the HSA-rtTA mouse could be used to study how the early ( $\sim 24 \mathrm{~h})$ activation of Akt1 (using the tetracycline-responsive, constitutively active Akt1 mouse, TRE-myrAkt1) affects the hypertrophic response in skeletal muscle as Akt1 is typically not activated until $48 \mathrm{~h}[16,17]$.

In contrast to the strong rtTA expression in hind limb muscles, there was modest, but detectable, expression of rtTA mRNA in the diaphragm. We do not know the reason why rtTA expression is lower in the diaphragm compared to hind limb muscles, but it may reflect a limitation of the HSA promoter to drive high levels of expression in the diaphragm. We also found comparatively lower expression of Cre in the diaphragm of the HSA-CreER mouse, consistent with the idea that the HSA promoter is not as robust in the diaphragm as it is in other muscles. Despite low rtTA expression, we still observed $\sim 60 \%$ GFP labeling of diaphragm myonuclei after only $24 \mathrm{~h}$ of doxycycline treatment. This result indicates the HSA-rtTA mouse is a useful tool for investigators studying the diaphragm.

The HSA-rtTA transgenic mouse allows for inducible, myofiber-specific gene expression in both slow- and fast-twitch muscles. The HSA-rtTA mouse will provide researchers with a powerful tool to reversibly induce gene expression in an effort to better understand skeletal muscle biology. The HSA-rtTA mouse will be freely available upon request.

\footnotetext{
Abbreviations

dox: Doxycycline; EDL: Extensor digitorum longus; GFP: Green fluorescent protein; $\mathrm{H} 2 \mathrm{~B}$ : Histone $\mathrm{H} 2 \mathrm{~B}$; HSA: Human skeletal muscle a-actin; IHC: Immunohistochemistry; rtTA: Reverse tetracycline transactivator; TA: Tibialis anterior; Tet-ON/OFF: Tetracycline-responsive system
}

Funding

This work was supported by NIH grants AG049806 and AR060701 to JJM and CAP and AR071753 to KAM

\section{Availability of data and materials}

The datasets used and/or analyzed during the current study are available from the corresponding author on request. The HSA-rtTA mouse is available upon request.

\section{Declarations}

N.A.

\section{Authors' contributions}

JJM cloned the transgene. MI, DAE, YW, CMD, KAM, IJ, CBM, CAP, and JJM assisted in developing the study design and characterizing the mouse. DAE and JJM wrote the manuscript. All authors read and approved the final manuscript.

\section{Ethics approval and consent to participate}

All animal procedures were conducted in accordance with institutional guidelines for the care and use of laboratory animals as approved by the Animal Care and Use Committee of the University of Kentucky.

\section{Consent for publication}

N.A

\section{Competing interests}

The authors declare that they have no competing interests.

\section{Publisher's Note}

Springer Nature remains neutral with regard to jurisdictional claims in published maps and institutional affiliations.

\section{Author details}

${ }^{1}$ The Center for Muscle Biology, University of Kentucky, Lexington, KY 40536, USA. ${ }^{2}$ Department of Rehabilitation Sciences, College of Health Sciences, University of Kentucky, Lexington, KY 40536, USA. ${ }^{3}$ Department of Physiology, College of Medicine, University of Kentucky, 800 Rose Street, Medical Science Building, Rm: MS-607A, Lexington, KY 40536, USA. ${ }^{4}$ Department of Rehabilitation, Faculty of Health Sciences, Nihon Fukushi University, 26-2 Higashihaemi-cho, Handa 475-0012, Japan.

Received: 27 June 2018 Accepted: 16 October 2018

Published online: 27 October 2018

\section{References}

1. Furth PA, St Onge L, Boger H, Gruss P, Gossen M, Kistner A, et al. Temporal control of gene expression in transgenic mice by a tetracycline-responsive promoter. Proc Natl Acad Sci U S A. 1994;91(20):9302-6.

2. Das AT, Tenenbaum L, Berkhout B. Tet-On systems for doxycycline-inducible gene expression. Curr Gene Ther. 2016;16(3):156-67.

3. Ghersa P, Gobert RP, Sattonnet-Roche P, Richards C, Pich EM, Van Huijsduijnen RH. Highly controlled gene expression using combinations of a tissue-specific promoter, recombinant adenovirus and a tetracyclineregulatable transcription factor. Gene Ther. 1998:5(9):1213.

4. Grill MA, Bales MA, Fought AN, Rosburg KC, Munger SJ, Antin PB. Tetracycline-inducible system for regulation of skeletal muscle-specific gene expression in transgenic mice. Transgenic Res. 2003;12(1):33-43.

5. Tumbar T, Guasch G, Greco V, Blanpain C, Lowry WE, Rendl M, et al. Defining the epithelial stem cell niche in skin. Science. 2004;303(5656):359-63.

6. McCarthy JJ, Srikuea R, Kirby TJ, Peterson CA, Esser KA. Inducible Cre transgenic mouse strain for skeletal muscle-specific gene targeting. Skelet Muscle. 2012;2(1):8.

7. Murach KA, White SH, Wen $\mathrm{Y}$, Ho A, Dupont-Versteegden EE, McCarthy JJ, et al. Differential requirement for satellite cells during overload-induced muscle hypertrophy in growing versus mature mice. Skelet Muscle. 2017:7(1):14

8. Zhou X, Vink M, Klaver B, Berkhout B, Das AT. Optimization of the Tet-On system for regulated gene expression through viral evolution. Gene Ther. 2006;13(19):1382-90 
9. Muscat GE, Kedes L. Multiple 5'-flanking regions of the human alpha-skeletal actin gene synergistically modulate muscle-specific expression. Mol Cell Biol. 1987;7(11):4089-99.

10. Izumiya Y, Hopkins T, Morris C, Sato K, Zeng L, Viereck J, et al. Fast/Glycolytic muscle fiber growth reduces fat mass and improves metabolic parameters in obese mice. Cell Metab. 2008;7(2):159-72.

11. Schuler M, Ali F, Metzger E, Chambon P, Metzger D. Temporally controlled targeted somatic mutagenesis in skeletal muscles of the mouse. Genesis. 2005;41(4):165-70

12. Nishijo K, Hosoyama T, Bjornson CR, Schaffer BS, Prajapati SI, Bahadur AN, et al. Biomarker system for studying muscle, stem cells, and cancer in vivo. FASEB J. 2009;23(8):2681-90.

13. Lepper C, Conway SJ, Fan CM. Adult satellite cells and embryonic muscle progenitors have distinct genetic requirements. Nature. 2009;460(7255):627-31.

14. Murphy MM, Lawson JA, Mathew SJ, Hutcheson DA, Kardon G. Satellite cells, connective tissue fibroblasts and their interactions are crucial for muscle regeneration. Development. 2011;138(17):3625-37.

15. Lee SJ, Huynh TV, Lee YS, Sebald SM, Wilcox-Adelman SA, Iwamori N, et al. Role of satellite cells versus myofibers in muscle hypertrophy induced by inhibition of the myostatin/activin signaling pathway. Proc Natl Acad Sci U S A. 2012;109(35):E2353-60.

16. Shiojima I, Sato K, Izumiya Y, Schiekofer S, Ito M, Liao R, et al. Disruption of coordinated cardiac hypertrophy and angiogenesis contributes to the transition to heart failure. J Clin Invest. 2005;115(8):2108-18.

17. Miyazaki M, McCarthy JJ, Fedele MJ, Esser KA. Early activation of mTORC1 signalling in response to mechanical overload is independent of phosphoinositide 3-kinase/Akt signalling. J Physiol. 2011;589(Pt 7):1831-46.

Ready to submit your research? Choose BMC and benefit from:

- fast, convenient online submission

- thorough peer review by experienced researchers in your field

- rapid publication on acceptance

- support for research data, including large and complex data types

- gold Open Access which fosters wider collaboration and increased citations

- maximum visibility for your research: over $100 \mathrm{M}$ website views per year

At $\mathrm{BMC}$, research is always in progress.

Learn more biomedcentral.com/submissions 